\title{
26 Research Square \\ Chronic low back pain changes in trunk controls of gait
}

Yuki Nishi ( $\nabla$ y.nishi.pt@gmail.com )

Graduate School of Health Science, Kio University

Hayato Shigetoh

Graduate School of Health Science, Kio University

Ren Fujii

Graduate School of Health Science, Kio University

Michihiro Osumi

Graduate School of Health Science, Kio University

Shu Morioka

Graduate School of Health Science, Kio University

\section{Research Article}

Keywords: chronic low back pain, daily-living gait, variability indexes, stability indexes, inertial sensors

Posted Date: June 3rd, 2021

DOI: https://doi.org/10.21203/rs.3.rs-127066/v2

License: (c) (1) This work is licensed under a Creative Commons Attribution 4.0 International License.

Read Full License 


\section{Abstract}

This study investigated the impact of environment in trunk controls of gait among individuals with CLBP. CLBP patients $(n=20)$ and healthy controls $(H C)$ with no LBP history $(n=20)$ attached an accelerometer to the low back and performed gait in laboratory and daily-living settings. We calculated stride-to-stride standard deviation (SD) and multiscale sample entropy (MSE) as "gait variability" in both the anteriorposterior (AP) and medial-lateral (ML) directions. In repeated-measures ANOVA, SD was affected by environment in the AP direction and by group and environment in the ML direction. MSE showed no effect in the AP direction and both effects in the ML direction. These change of trunk motor control by LBP have a relation to pain intensity, fear of movement and/or RMDQ in the daily-living but not laboratory settings. Evaluation of adaptation to environment in CLBP will enhance our understanding of changes in sensorymotor control and contribute to optimal management of such patients.

\section{Introduction}

Individuals with chronic low back pain (CLBP) are known to have characteristic spatiotemporal gait parameters including step length, cadence and stride-to-stride variability ${ }^{1-5}$. In addition, they frequently present with impaired lumbar movements such as a decline movement velocity and a reduced range of movement ${ }^{6}$. In general, motor control is affected among individuals with LBP in the way that Hodges propose that changes occur at multiple levels of the motors system. Pain and/or pain-related fear reportedly alters motor variability and trunk stabilization as motor system adaptations in individuals with $\mathrm{LBP}^{7}$. However, this effect has not been clarified in relation to gait. Gait variability is a reflection of the multiple degrees of freedom of the sensory-motor system ${ }^{8}$. Both standard deviation (SD), which is one of the classical linear estimates, and multiscale sample entropy (MSE), which is based on the quantification of complexity on multiple scales of time series, have been used to evaluate gait variability ${ }^{9-11}$.

Previous studies employing a single wearable sensor to evaluate gait reported that variability and/or stability of gait are affected more in the daily-living than the laboratory or clinical setting ${ }^{14-16}$. In particular, healthy young adults routinely vary their motor patterns to maintain stability in unstructured environments ${ }^{16}$. Measuring gait in daily living reflects habitual gait performance ${ }^{17,18}$; thus, recording daily-living gait has the potential benefits of detecting gait in unsupervised and uncontrolled conditions 19,20 . Moreover, gait in unstructured daily living is more difficult to predict and hence prone to disturbance than that in structured clinical settings. Compared to healthy adults, individuals with CLBP may show increased gait variability and decreased gait stability in daily living secondary to changes in trunk motor control related to pain and/or pain-related fear.

Therefore, this study aimed to use measures of SD, MSE to explore the impact of environment and pathology by analysing differences of trunk variability and stability of gait between individuals with LBP and healthy controls in both laboratory and daily-living environments. In addition, we investigated the relationship between trunk motor controls of gait and pain and/or fear. As our hypotheses, we posited 
that between-group and/or between-environments differences would be more apparent in CLBP, and that trunk motor controls of gait would show a relationship to symptoms of CLBP.

\section{Subjects And Methods}

\subsection{Subjects and assessment}

We recruited twenty healthy participants (mean age $56.75 \pm 9.43$ years) from our laboratory's geographic region using flyers, and twenty chronic low back pain patients (mean age $54.05 \pm 10.76$ years) from an orthopedic clinic. The inclusion criteria for the experimental group were as follows: a low back pain (LBP) duration of $>3$ months; a score of $\geq 1$ on an 11-point numeric rating scale (NRS, $0-10)$ for pain intensity; sufficient walking ability to travel to our laboratory without assistance. The exclusion criteria were as follows: previous spinal surgery (fusion, instrumentation, or discectomy), lumbar infiltration in the last 6 months, serious spinal pathology (cancer, inflammatory arthropathy, or acute vertebral fracture), spinal deformities, leg pain (e.g. hip/knee $O A$ ), a diagnosis of neurological disease, and a stage of $\geq 2$ on a functional assessment staging test of Alzheimer's disease. The study protocol conformed to the Declaration of Helsinki. Before participating, each participant provided written informed consent. This study was approved by the Ethics Committee of Kio University Health Science Graduate School (approval no. H30-20).

\subsection{Data collection in the laboratory and daily-living}

In the laboratory, each CLBP patient was instructed to fill out 3 questionnaires about LBP. Average pain intensity over time was assessed on an 11-point numerical rating scale (NRS: $0=$ no pain and $10=$ highest possible degree of pain). Kinesiophobia was assessed using the 11-item version of the Tampa Scale for Kinesiophobia (TSK-11:11 = no fear of movement and $44=$ highest fear of movement) ${ }^{21}$, and physical disability caused by LBP was assessed on Roland-Morris Disability Questionnaire (RMDQ: $0=$ no physical disability and $24=$ worse physical function) ${ }^{22}$.

Next, each participant was asked to wear a single wearable tri-axial accelerometer (Axivity AX3, York, UK) on the fifth lumbar vertebra (L5). The device was programmed to capture data at $100 \mathrm{~Hz}$ and at a range of $\pm 8 \mathrm{~g}^{23}$. In the laboratory environment, participants walked over 60 seconds at their preferred speed around an indoor track ( $30 \mathrm{~m} / \mathrm{loop}$ ). After undergoing the clinical assessment, participants were asked to wear a single wearable sensor for three days in a daily living environment; i.e., to continue their daily activities as usual ${ }^{24}$.

\subsection{Data processing and analysis}

\subsubsection{Description of Acceleration-Derived Variables}

All data analysis relating to the raw acceleration signals was performed using custom-made MATLAB R2019b (MathWorks BV, USA). The algorithm and data segmentation techniques applied to the 
accelerometer data have already been described in full ${ }^{23,25}$. In brief, the vertical acceleration underwent continuous wavelet transformation to estimate the initial contact and final contact in the gait cycle ${ }^{25}$. To ensure that steady-state gait was analyzed, the initial and final three steps were removed from the data captured over 60 seconds. In a daily living environment, A mean of 15.75 walking epochs (range: 6 to 32 ) with duration over 60 seconds were identified for each of the healthy participants. Similarly, a mean of 15.15 walking epochs (range: 5 to 26) with duration over 60 seconds were identified for each of the CLBP patients. Prior to the calculation of additional variables, the acceleration signals were realigned to the earth's gravitational constant, and a low-pass Butterworth filter with a cut-off frequency of $20 \mathrm{~Hz}$ was applied ${ }^{26,27}$. The following variables were calculated using the accelerations of anterior-posterior (AP) and medial-lateral $(\mathrm{ML})$ directions since directional control of movement is performed independently ${ }^{28}$.

\subsubsection{Trunk variability of gait}

Standard Deviation (SD)

To compute stride-to-stride variability of gait, trunk acceleration during each stride was time-normalized (0-100\%). At each of the 101 normalized time points, the SDs of AP and ML trunk acceleration were calculated. Next, the average SD of these 101 SDs was calculated ${ }^{9}$.

Multiscale sample entropy

Multiscale entropy (MSE) is a nonlinear analysis to quantify the complexity of biological signals based on the dynamical system theory that the dynamics of motor behavior results from interaction between the nervous system, body and the environment in the performance of a particular task on multiple spatiotemporal scales ${ }^{10,11}$. This measure is more sensitive than linear variability and can serve for investigating complexity in motor control ${ }^{29}$. This index aims at quantifying the predictability of patterns, a time series containing many repetitive patterns (i.e., one that is more predictable) would have a relatively small MSE, whereas a less predictable process would have a higher MSE and smaller regularity ${ }^{10}$. A range of $\tau$-values (from 1 to 6 ) were used to analyse entropy at different time scales, while preserving the characteristics of the original time series. The length of sequences to be compared, $\mathrm{m}$, was fixed at 2 and the tolerance for accepting matches, r, at $0.2^{30,31}$.

\subsection{Statistical analyses}

We used the software program $\mathrm{R}$ (version 3.4.1) for all statistical analyses. We compared the age and gender distribution between the HC and CLBP groups by using the t-test and chi-squared test, respectively. For trunk motor controls of gait, a 2' 2 [Environment (Lab/daily living) ' Group (HC/CLBP)] repeated measures analysis of variance (RM-ANOVA) was conducted in the ML and AP directions. Partial eta square $\left(n_{p}{ }^{2}\right)$ values were calculated to identify effect sizes. Independent and dependent t tests were used for post hoc analysis when significant group by condition interactions were identified. Independent $t$ tests were used to compare demographics between groups, and $d$ values were calculated to indicate 
effect sizes. In addition, the associations between trunk motor controls of gait and symptoms of CLBP were evaluated using Pearson product-moment correlation coefficients. Analysis was corrected for multiple comparisons using Holm corrections ${ }^{36}$.

\section{Results}

The RM-ANOVA of MSE revealed no significant main effects for Environment or Group. In the ML direction, the RM-ANOVA of the SD revealed significant main effects for Group and Environment. An MSE of $\tau=1$ showed significant main effects for Group but not for Environment. An MSE of $\tau \geq 2$ showed significant main effects for Environment and Group, and revealed that the interaction of Environment ' Group was significant. The post hoc analyses were performed by MSE at $\tau \geq 2$ for ML and revealed that the occurrence of MSE at $\tau \geq 2$ in daily living was significantly higher than that in the lab for the CLBP group. In addition, MSE at $\tau \geq 4$ in CLBP was significantly higher than that of the controls in the lab setting, while MSE at $\tau \geq 2$ in CLBP was significantly higher than that of the controls in the daily-living setting.

\section{Discussion}

In this study, we used a single wearable sensor, placed on the lower back, to assess trunk variability and stability of gait in individuals with chronic low back pain (CLBP) and healthy controls (HC) in laboratory and daily-living environments. We hypothesized that individuals with CLBP have increased gait variability in daily living compared to HC.

Our results were as follows: (1) the standard deviation (SD) of trunk acceleration (i.e. trunk variability) in the CLBP group was high regardless of environment in the Anterior-Posterior (AP) direction and high but affected by environment in the Medial-Lateral (ML) direction. (2) Multiscale sample entropy (MSE) (i.e. complexity) was not affected by CLBP status or by environment in the AP direction but was high in the CLBP group and affected by environment in the ML direction. In addition, the MSE at $\tau \geq 2$ was higher in the CLBP group in the daily-living setting. (3) These changes of trunk motor control by CLBP have a relation to pain intensity and/or fear of movement and/or ADL deficits in the daily-living but not lab setting. Overall, our results supported our hypothesis that individuals with CLBP would show increased trunk variability and decreased stability of gait, but the AP and ML directions showed differences in these trends of trunk variability of gait.

Trunk variability of gait was increased in the daily-living setting in the CLBP group. Gait in the unstructured environment of daily living may be more difficult to predict and prone to perturbations than in structured clinical settings, because the unstructured environment reinforces the need to adapt movements with a high degree of freedom (DOF). On the other hand, gait in daily living can be interpreted as a cognitively dual task, and daily activities often incur a heavier cognitive load. It was previously reported that those with CLBP showed increased trunk variability in dual-task walking by loss of automatism of the sensory-motor control system due to chronic pain ${ }^{4}$. In the perspective of spinal 
structure, the lumbar spine has the largest DOF number and therefore greater problems with controlling position, especially with high loads and in unpredictable settings ${ }^{37}$. The loose control (i.e. increased trunk variability) can cause LBP, as large displacements after trunk perturbations are associated with LBP and failures of muscular control over the spine result in large tissue strains ${ }^{37-39}$.

The increased complexity of gait in CLBP that we showed in this study is consistent with the results of previous studies ${ }^{40-42}$. It also has been reported that gait complexity increases in lumbar spinal stenosis patients and is directly related to the intensity of low back pain ${ }^{43}$. Change in the neuromuscular control and/or pain may compel participants to compensate by adjusting their gait pattern. Also, the effect of environment or group increases with time delay $\tau$, in agreement with the previous research ${ }^{44,45}$. In particular, a $\tau$ higher than 4 means that frequencies below $10-16 \mathrm{~Hz}$ contribute the most in characterizing the automaticity of the human gait ${ }^{46}$. In addition to the effects of environment and CLBP, individuals with CLBP may lose gait automatism and require high cognitive attention-in structured as well as unstructured environments. From the above, although individuals with CLBP perform compensatory gait loosening strategies as an adaptation to the environment, they may simultaneously increase their cognitive load, leading to chronicity of pain 4,5 .

For both stability and variability of gait, data in the AP and ML directions showed different trends. Specifically, in the AP direction, stability of gait was impaired, and the variability was not affected by environmental changes; in the ML direction, the variability was affected by environment and CLBP, but the stability was maintained. A previous study reported change in the trunk variability of gait as an adaptation to environment performed to maintain trunk stability ${ }^{16}$. A decrease in stability in the AP direction, even though the variability does not change, may indicate less compensation or inadequate compensation ${ }^{29}$. Dysfunction of the agonist/ antagonist in the lumbar extension also impedes motor adaptation of perturbation ${ }^{54,55}$. Therefore, in the AP direction, individuals with CLBP may have difficulty adapting to the environment, resulting in decreased stability in unstructured daily living. On the other hand, in the ML direction, the stability of gait can be maintained by adaptations to the environment via changes in variability. These results were associated with subjective intensity of pain and fear of movement and were more pronounced in daily living. In addition, trunk stability of gait in the AP direction was related to physical disability in daily living. Previous studies have shown that pain and/or fear promote changes in the sensory-motor control system and movement strategy ${ }^{56,57}$. Changes in gait control modified by pain and/or fear of movement may disturb a person's adaptation to their environment.

In terms of the present study's limitations, it should be considered that the sample size was relatively small, meaning that the results presented here should be interpreted with caution. In addition, because the questionnaires about pain and fear of movement were not assessed separately in the laboratory and daily living, they may have tended to reflect the pain experienced in the daily-living setting. 
In conclusion, we revealed that individuals with CLBP undergo changes in trunk variability of gait depending on the environment and that these changes are related to pain and fear, quality of life. Interestingly, in the AP direction, stability of gait was impaired, and the variability was not affected by environmental changes; in the ML direction, the variability was affected by environment, but the stability was maintained. Although the underlying causes of these different adaptations of gait variability by direction remain to be investigated, we suspect changes in muscle and/or sensory-motor control system. Evaluation of such adaptations to environment in CLBP will enhance our understanding of changes in sensory-motor control by pain and/or fear, and provide information for clinical decision-making and intervention development.

\section{Declarations}

\section{Funding sources:}

This research was not supported by a grant.

\section{Conflict of interest statement:}

The authors have no conflicts of interest to declare.

\section{References}

1. Keefe, F. J. \& Hill, R. W. An objective approach to quantifying pain behavior and gait patterns in low back pain patients. Pain 21, 153-161 (1985).

2. Lamoth, C. J. C., Meijer, O. G., Daffertshofer, A., Wuisman, P. I. J. M. \& Beek, P. J. Effects of chronic low back pain on trunk coordination and back muscle activity during walking: Changes in motor control. Eur. Spine J. 15, 23-40 (2006).

3. Vogt, L., Pfeifer, K., Portscher, M. \& Banzer, W. Influences of nonspecific low back pain on threedimensional lumbar spine kinematics in locomotion. Spine (Phila. Pa. 1976). 26, 1910-1919 (2001).

4. Hamacher, D., Hamacher, D. \& Schega, L. A cognitive dual task affects gait variability in patients suffering from chronic low back pain. Exp. Brain Res. 232, 3509-3513 (2014).

5. Hamacher, D., Hamacher, D., Herold, F. \& Schega, L. Are there differences in the dual-task walking variability of minimum toe clearance in chronic low back pain patients and healthy controls? Gait Posture 49, 97-101 (2016).

6. Laird, R. A., Gilbert, J., Kent, P. \& Keating, J. L. Comparing lumbo-pelvic kinematics in people with and without back pain: A systematic review and meta-analysis. BMC Musculoskelet. Disord. 15, 1-13 (2014).

7. Hodges, P. W. Pain and motor control: From the laboratory to rehabilitation. J. Electromyogr. Kinesiol. 21, 220-228 (2011). 
8. Bruijn, S. M., Meijer, O. G., Beek, P. J. \& Van Dieen, J. H. Assessing the stability of human locomotion: A review of current measures. J. R. Soc. Interface 10, (2013).

9. Magnani, R. M. et al. Local dynamic stability and gait variability during attentional tasks in young adults. Gait Posture 55, 105-108 (2017).

10. Costa, M., Peng, C. K., Goldberger, A. L. \& Hausdorff, J. M. Multiscale entropy analysis of human gait dynamics. Phys. A Stat. Mech. its Appl. 330, 53-60 (2003).

11. Costa, M., Goldberger, A. L. \& Peng, C. K. Multiscale entropy analysis of biological signals. Phys. Rev. E - Stat. Nonlinear, Soft Matter Phys. 71, 1-18 (2005).

12. Kang, H. G. \& Dingwell, J. B. Intra-session reliability of local dynamic stability of walking. Gait Posture 24, 386-390 (2006).

13. Kao, P. C., Dingwell, J. B., Higginson, J. S. \& Binder-Macleod, S. Dynamic instability during post-stroke hemiparetic walking. Gait Posture 40, 457-463 (2014).

14. Ihlen, E. A. F., Weiss, A., Bourke, A., Helbostad, J. L. \& Hausdorff, J. M. The complexity of daily life walking in older adult community-dwelling fallers and non-fallers. J. Biomech. 49, 1420-1428 (2016).

15. Ihlen, E. A. F., van Schooten, K. S., Bruijn, S. M., Pijnappels, M. \& van Dieën, J. H. Fractional stability of trunk acceleration dynamics of daily-life walking: toward a unified concept of gait stability. Front. Physiol. 8, 1-15 (2017).

16. Tamburini, P. et al. Moving from laboratory to real life conditions: Influence on the assessment of variability and stability of gait. Gait Posture 59, 248-252 (2018).

17. De Bruin, E. D. et al. Validity and reliability of accelerometer-based gait assessment in patients with diabetes on challenging surfaces. J. Aging Res. 2012, (2012).

18. Lord, S., Galna, B. \& Rochester, L. Moving forward on gait measurement: Toward a more refined approach. Mov. Disord. 28, 1534-1543 (2013).

19. Weiss, A. et al. Does the evaluation of gait quality during daily life provide insight into fall risk? A novel approach using 3-Day accelerometer recordings. Neurorehabil. Neural Repair 27, 742-752 (2013).

20. Weiss, A., Herman, T., Giladi, N. \& Hausdorff, J. M. Objective assessment of fall risk in Parkinson's disease using a body-fixed sensor worn for 3 days. PLoS One 9, (2014).

21. Woby, S. R., Roach, N. K., Urmston, M. \& Watson, P. J. Psychometric properties of the TSK-11: A shortened version of the Tampa Scale for Kinesiophobia. Pain 117, 137-144 (2005).

22. Roland, M. O. The natural history of back pain. Practitioner vol. 227 1119-1122 (1983).

23. Del Din, S., Godfrey, A. \& Rochester, L. Validation of an Accelerometer to Quantify a Comprehensive Battery of Gait Characteristics in Healthy Older Adults and Parkinson's Disease: Toward Clinical and at Home Use. IEEE J. Biomed. Heal. Informatics 20, 838-847 (2016).

24. Van Schooten, K. S. et al. Assessing physical activity in older adults: Required days of trunk accelerometer measurements for reliable estimation. J. Aging Phys. Act. 23, 9-17 (2015). 
25. McCamley, J., Donati, M., Grimpampi, E. \& Mazzà, C. An enhanced estimate of initial contact and final contact instants of time using lower trunk inertial sensor data. Gait Posture 36, 316-318 (2012).

26. Buckley, C., Galna, B., Rochester, L. \& Mazzà, C. Quantification of upper body movements during gait in older adults and in those with Parkinson's disease: impact of acceleration realignment methodologies. Gait Posture 52, 265-271 (2017).

27. Moe-Nilssen, R. A new method for evaluating motor control in gait under real-life environmental conditions. Part 2: Gait analysis. Clin. Biomech. 13, 328-335 (1998).

28. Bruijn, S. M. \& Van Dieën, J. H. Control of human gait stability through foot placement. J. R. Soc. Interface 15, (2018).

29. Stergiou, N. \& Decker, L. M. Human movement variability, nonlinear dynamics, and pathology: Is there a connection? Hum. Mov. Sci. 30, 869-888 (2011).

30. Pincus, S. M. Approximate entropy as a measure of system complexity. Proc. Natl. Acad. Sci. U. S. A. 88, 2297-2301 (1991).

31. Richman, J. S., Randall Moorman, J., Randall, J. \& Physi, M. Downloaded from www.physiology.org/journal/ajpheart by \$\{individualUser. Am J Physiol Hear. Circ Physio/ 278, 2039-2049 (2000).

32. Dingwell, J. B. \& Marin, L. C. Kinematic variability and local dynamic stability of upper body motions when walking at different speeds. J. Biomech. 39, 444-452 (2006).

33. Mehdizadeh, S. The largest Lyapunov exponent of gait in young and elderly individuals: A systematic review. Gait Posture 60, 241-250 (2018).

34. England, S. A. \& Granata, K. P. The influence of gait speed on local dynamic stability of walking. Gait Posture 25, 172-178 (2007).

35. Rosenstein, M. T., Collins, J. J. \& De Luca, C. J. A practical method for calculating largest Lyapunov exponents from small data sets. Phys. D Nonlinear Phenom. 65, 117-134 (1993).

36. Holm, S. Board of the Foundation of the Scandinavian Journal of Statistics A Simple Sequentially Rejective Multiple Test Procedure A Simple Sequentially Rejective Multiple Test Procedure. Source Scand. J. Stat. Scand J Stat. 6, 65-70 (1979).

37. Panjabi, M. M. The stabilizing system of the spine: Part I. function, dysfunction, adaptation, and enhancement. Journal of Spinal Disorders vol. 5 383-389 (1992).

38. Cholewicki, J. et al. Delayed trunk muscle reflex responses increase the risk of low back injuries. Spine (Phila. Pa. 1976). 30, 2614-2620 (2005).

39. Van Dieën, J. H., Peter Reeves, N., Kawchuk, G., Van Dillen, L. R. \& Hodges, P. W. Motor control changes in low back pain: Divergence in presentations and mechanisms. J. Orthop. Sports Phys. Ther. 49, 370-379 (2019).

40. Arif, M., Ohtaki, Y. \& Nagatomi, R. Estimation of the effect of cadence on gait stability in young and elderly people using approximate entropy technique. Meas. Sci. 4, 29-40 (2004). 
41. Karmakar, C. K., Khandoker, A. H., Begg, R. K., Palaniswami, M. \& Taylor, S. Understanding ageing effects by approximate entropy analysis of gait variability. Annu. Int. Conf. IEEE Eng. Med. Biol. Proc. 1965-1968 (2007) doi:10.1109/IEMBS.2007.4352703.

42. Lamoth, C. J. C., Ainsworth, E., Polomski, W. \& Houdijk, H. Variability and stability analysis of walking of transfemoral amputees. Med. Eng. Phys. 32, 1009-1014 (2010).

43. Papadakis, N. C. et al. Gait variability measurements in lumbar spinal stenosis patients: Part A. Comparison with healthy subjects. Physiol. Meas. 30, 1171-1186 (2009).

44. Bisi, M. C., Riva, F. \& Stagni, R. Measures of gait stability: Performance on adults and toddlers at the beginning of independent walking. J. Neuroeng. Rehabil. 11, 1-9 (2014).

45. Riva, F., Toebes, M. J. P., Pijnappels, M., Stagni, R. \& van Dieën, J. H. Estimating fall risk with inertial sensors using gait stability measures that do not require step detection. Gait Posture 38, 170-174 (2013).

46. Bisi, M. C. \& Stagni, R. Complexity of human gait pattern at different ages assessed using multiscale entropy: From development to decline. Gait Posture 47, 37-42 (2016).

47. Tong, M. H. et al. Is There a Relationship Between Lumbar Proprioception and Low Back Pain? A Systematic Review With Meta-Analysis. Arch. Phys. Med. Rehabil. 98, 120-136.e2 (2017).

48. Sjölander, P., Johansson, H. \& Djupsjöbacka, M. Spinal and supraspinal effects of activity in ligament afferents. J. Electromyogr. Kinesiol. 12, 167-176 (2002).

49. Mok, N. W. \& Hodges, P. W. Movement of the lumbar spine is critical for maintenance of postural recovery following support surface perturbation. Exp. Brain Res. 231, 305-313 (2013).

50. Ross, G. B., Mavor, M., Brown, S. H. M. \& Graham, R. B. The Effects of Experimentally Induced Low Back Pain on Spine Rotational Stiffness and Local Dynamic Stability. Ann. Biomed. Eng. 43, 21202130 (2015).

51. Lamoth, C. J. C. et al. Pelvis-thorax coordination in the transverse plane during walking in persons with nonspecific low back pain. Spine (Phila. Pa. 1976). 27, 92-99 (2002).

52. van den Hoorn, W., Hug, F., Hodges, P. W., Bruijn, S. M. \& van Dieën, J. H. Effects of noxious stimulation to the back or calf muscles on gait stability. J. Biomech. 48, 4109-4115 (2015).

53. Dingwell, J. B., Cusumano, J. P., Cavanagh, P. R. \& Sternad, D. Local dynamic stability versus kinematic variability of continuous overground and treadmill walking. J. Biomech. Eng. 123, 27-32 (2001).

54. Vogt, L., Pfeifer, K. \& Banzer, W. Neuromuscular control of walking with chronic low-back pain. Man. Ther. 8, 21-28 (2003).

55. Ghamkhar, L. \& Kahlaee, A. H. Trunk Muscles Activation Pattern During Walking in Subjects With and Without Chronic Low Back Pain: A Systematic Review. PM R 7, 519-526 (2015).

56. Karos, K. et al. Fear of pain changes movement: Motor behaviour following the acquisition of painrelated fear. Eur. J. Pain (United Kingdom) 21, 1432-1442 (2017). 
57. Nishi, Y., Osumi, M., Nobusako, S., Takeda, K. \& Morioka, S. Avoidance Behavioral Difference in Acquisition and Extinction of Pain-Related Fear. Front. Behav. Neurosci. 13, 1-10 (2019).

\section{Tables}

Due to technical limitations, table 1 and table 2 are only available as a download in the Supplemental Files section.

\section{Supplementary Files}

This is a list of supplementary files associated with this preprint. Click to download.

- Table1.xlsx

- Table2.xlsx 